\title{
APORTACIONES DEL PARADIGMA DE RESILIENCIA A LA ACCIÓN SOCIOEDUCATIVA. EL CASO DEL CENTRO DE MENORES EXTRANJEROS NO ACOMPAÑADOS ZABALOETXE
} RESILIENCE PARADIGM'S CONTRIBUTIONS TO SOCIO EDUCATIONAL WORK. THE CASE OF THE CENTER ZABALOETXE FOR UNACCOMPANIED MIGRANT CHILDREN CONTRIBUIÇÕES DO PARADIGMA DA RESILIÊNCIA NA AÇÃO EDUCATIVA. O CASO DO CENTRO “ZABALOETXE” PARA MENORES NÃO ACOMPANHADOS

PALABRAS CLAVE: resiliencia afectividad protección de menores educador social tutor de resiliencia

\footnotetext{
Fecha de recepción del artículo: 13.XI.2014

Fecha de revisión del artículo: 28.1.2015
} Fecha de aceptación final: 25.V.2015
RESUMEN: En este artículo se realiza un acercamiento a la resiliencia y la afectividad como elementos clave en la intervención socioeducativa. Consta de una fundamentación teórica y una investigación cualitativa que se centra en la intervención del educador y la educadora y su potencialidad como tutor y tutora de resiliencia en un centro residencial de menores extranjeros no acompañados. Desde una perspectiva cualitativa se han utilizado como técnicas de recogida de datos la observación participante y nueve entrevistas con 3 educadoras y 6 educadores y el coordinador del Servicio. El análisis e interpretación de los resultados proporcionan evidencias relacionadas con la detección de factores de riesgo y protección, las características de la intervención educativa en la fase de acogida, la afectividad y el ambiente como claves en el tiempo de representación y relación, y por último sobre las bases socioeducativas de la acción tutorial resiliente. Algunas de las conclusiones teniendo en cuenta los resultados de la investigación son: que la resiliencia comparte con la educación un factor determinante como es la importancia de la calidad del entorno humano y las interacciones que en este se dan, y que en la medida que se crea un vínculo educativo hay espacio para la estimulación de la misma. También se plantea la necesidad de seguir investigando en este ámbito relacionado con la Resilencia y la Educación Social.

ABSTRACT: In this article it has been carried out an approach to resilience and affectivity as key elements to socio-educational intervention. Its theoretical and qualitative research are based on the intervention of the educator and his ability as a resilience tutor within a center for unaccompanied migrant children. From a qualitative perspective, participant observation and 9 interviews held with 3 female and 6 male educators had been used. The analysis and interpretation of the results show some evidences linked to the detection of risk factors and protection, characteristics of the educational intervention in the reception phase, and the environment as key issues in the period of representation and 


\begin{tabular}{|c|c|}
\hline & $\begin{array}{l}\text { relation, and finally on the socio-educative bases of the resilience teaching action. Some } \\
\text { of the conclusions take in account the results of this investigation are the following: re- } \\
\text { silience shares with education a key factor such as the importance of the quality of the hu- } \\
\text { man environment and the interactions manifested within this last one, and as an educational } \\
\text { bond is created, there is a space for its own stimulation. It is also set out the necessity of } \\
\text { continuing with the research within this field linked to the resilience and the social edu- } \\
\text { cation. }\end{array}$ \\
\hline $\begin{array}{l}\text { PALAVRAS-CHAVE: } \\
\text { resiliência } \\
\text { carinho } \\
\text { proteção à criança } \\
\text { educador social } \\
\text { tutor de resiliência }\end{array}$ & $\begin{array}{l}\text { RESUMO: Este artigo descreve uma abordagem para a resiliência e emoções como ele- } \\
\text { mentos-chave da intervenção educativa realizada. Consiste de uma base teórica e uma pes- } \\
\text { quisa qualitativa que incide sobre a intervenção do professor e educador e seu potencial } \\
\text { como tutor e tutor de resiliência numa instalação residencial para crianças desacompan- } \\
\text { hadas. A partir de uma perspectiva qualitativa, têm sido utilizadas, como técnicas de coleta } \\
\text { de dados, a observação participante e nove entrevistas com educadores e coordenadora } \\
\text { do Serviço. A análise e interpretação dos resultados fornecem evidências relacionadas com } \\
\text { a deteção de fatores de risco e características de proteção da intervenção educacional na } \\
\text { fase de recepção, as emoções e o meio ambiente como representação de chave no tempo } \\
\text { e no relacionamento e, finalmente, com base no tutorial sócio resiliente. Alguns dos resul- } \\
\text { tados, tendo em conta os resultados da pesquisa, são: que as quotas de resiliência com a } \\
\text { educação como fator determinante é a importância da qualidade do ambiente humano e } \\
\text { as interações que ocorrem neste; e que, quando é criado um vínculo educativo, há espaço } \\
\text { para estimular o mesmo. É também apontada a necessidade de mais investigação nesta } \\
\text { área relacionada com Resiliência e Educação Social. }\end{array}$ \\
\hline
\end{tabular}

\section{O. Introducción}

- La libertad, el trabajo, la buena suerte, todo lo dispone Dios y su profeta.

-Pero ¿por qué no nos da Dios la misma suerte que a

los demás? -pregunté a mi madre.

-Solo Dios lo sabe. Nosotros no sabemos nada y tampoco debemos preguntar.

Mohamed Chukri (2000). El pan desnudo.

Mohamed Chukri hizo de su sufrimiento de infancia una magnífica obra literaria reflejada en El pan desnudo (2000). Seguramente nadie hubiera creído que aquel joven analfabeto que deambulaba por Tánger inmerso en la más absoluta pobreza y marginalidad acabaría traduciendo al árabe obras de Becker o García Lorca y siendo uno de los referentes de la literatura árabe contemporánea. Reparar en la obra y vida de Mohamed Chukri nos acerca a la temática de este trabajo de investigación. Por un lado, porque él, lo mismo que la mayoría del colectivo relacionado con este estudio, fue un joven marroquí desarrollado en la adversidad y, por otro, porque logró retomar el camino de su existencia y hacer de esa adversidad algo útil que ofrecer, lo que denota factores propios del concepto objeto de este artículo: el proceso de resiliencia.

\section{Marco teórico}

\subsection{La resiliencia como proceso que se desarrolla en el entorno afectivo y social}

El término resiliencia proveniente del mundo de la física, expresa la capacidad que tienen todos los ma- teriales a resistir la presión, doblarse con flexibilidad, recobrar su forma original, su capacidad de resistencia al choque, y no deformarse ante presiones y fuerzas externas (De Pedro \& Muñoz, 2005). Tiene su origen en el latín, resilio que significa rebotar, volver de un salto, volver atrás, resaltar (Kotliarenco, Cáceres \& Fontecilla, 1992). Fue J. Bolwby desde el campo de la psicología el primero en usar la palabra resiliencia enfatizando el papel del vínculo afectivo en la génesis de ésta, definiéndola como "resorte moral, cualidad de una persona que no se desanima, que no se deja abatir" (Bolwby, 1992, citado por De Pedro \& Muñoz, 2005).

El origen del término en las ciencias sociales es atribuido a Enmy Werner (2007), quien tras un estudio longitudinal observó que niños que habían vivido en condiciones fuertemente adversas fueron capaces de desarrollarse de manera exitosa y feliz. Estas personas fueron llamadas "resilientes" (Barranco, 2008). En este sentido, Munist y otros autores (1998, p.14) definen a las personas resilientes como:

“(...) aquellos que al estar insertos en una situación de adversidad, es decir, al estar expuestos a un conglomerado de factores de riesgo, tienen la capacidad de utilizar aquellos factores protectores para sobreponerse a la adversidad, crecer y desarrollarse adecuadamente, llegando a madurar como seres adultos competentes, pese a los pronósticos desfavorables".

Hoy es aceptado por diferentes autores (Villalba, 2013; Margalit \& Idan, 2004; Reich, Zautra, \& Hall, 2010; Munist, Suarez \& Suarez, 2010) la resiliencia como un paradigma en la acción social y educativa que implica una nueva mirada a las dificultades y necesidades hu- 
manas enfatizando la potencialidades y las fortalezas que tienen todas las personas para hacerlas frente así como la importancia de la calidad relacional en su desarrollo. En la última década es destacable el ascendente número de investigaciones y publicaciones relacionadas con la resiliencia y la acción socioeducativa (Villalba, 2003, 2011; Ungar, 2004; Drapeau, SaintJacques, Lepine, Begin, \& Bernard, 2007; Muñoz-Silva, 2012). En sus diferentes abordajes son numerosas y diferentes las definiciones de resiliencia recopiladas (Becoña, 2006; Fuentes, Medina, Van Barnebeld \& Escobar 2009; Kotliarenco, Caceres \& Fontecilla, 1997; O'Dougherty, Masten, \& Narayan, 2013; Poseck, Baquero \& Jimenez, 2006; Walsh, 2012) mostrando la difícil precisión y la amplitud del concepto. Es observada como resorte, habilidad, capacidad o cualidad pero siempre proveniente de procesos sociales e intrapsíquicos y no como elementos innatos; procesos que posibilitan una vida sana en un medio insano (Rutter, 1993, citado por Aguirre, 2004). La resiliencia se entiende como universal, no está en los seres excepcionales sino en todas las personas y en las variables naturales del entorno (Vanistandael \& Lecomté, 2002).

La resiliencia no es un catálogo de cualidades que pueda poseer un individuo sino un camino, un proceso que, desde el nacimiento hasta la muerte nos teje sin cesar uniéndonos a nuestro entorno (Cyrulnik, 2008). Como plantea este autor: "se trata de un proceso, de un conjunto de fenómenos armonizados en el que el sujeto se cuela en un contexto afectivo, social y cultural. La resiliencia es el arte de navegar en los torrentes" (Cyrulnik, 2008, p.212).

En la aparición de la resiliencia están presentes tanto factores de riesgo como de protección que ayudan a conseguir resultados positivos así como reducir o evitar los negativos (Becoña, 2006). Se entiende por factor de riesgo toda aquella característica individual, familiar o social que incrementa la posibilidad de sufrimiento, disfunción o desajuste y por factor protector toda característica, hecho o situación que mitiga el efecto de los factores de riesgo y eleva la capacidad para enfrentarse a circunstancias adversas (Pereira, 2007).

Desde la Pedagogía social el estudio que Boris Cyrulnik hace en su obra 'Los patitos feos. La resiliencia: una infancia infeliz no determina la vida' es muy a tener en cuenta. Continuamente enfatiza la importancia del entorno, políticas gubernamentales incluidas, y de las relaciones que se establecen en él, tanto para la evolución de los recursos internos que actúan como base en el proceso de resiliencia, como para la propia génesis y fortalecimiento de la misma. Este entorno afectivo y social tiene la capacidad de avivar o apagar las "llamas" de la resiliencia.

Cyrulinik (2008) profundiza en el proceso en el que se imbuyen las personas "heridas" pero resilientes y subraya la idea de que un mismo acontecimiento trau- matizante puede tomar diferentes direcciones en función del entorno afectivo e institucional que el contexto social disponga. El trauma, dice, es la asunción de la intersubjetividad y el desenlace del proceso de resiliencia, es la transformación del sufrimiento en una "obra de arte" o "una obra útil".

\subsection{Los eslabones de la resiliencia. El anda- miaje o complejo tejido de "los patitos feos"}

La resiliencia no es una progresión lineal y sin fallas (Vanistandael \& Lecomte, 2002), sino un proceso dependiente del entorno humano, en el que intervienen muchísimos factores y válido tanto para afrontar como para prevenir las adversidades. Somos vulnerables ante la fuerza de los eventos pero la resiliencia, como proceso, abre el campo de la creatividad y la libertad, y actúa como escudo protector (Grotberg, 2001; Muñoz \& De Pedro, 2005) siempre y cuando se cimiente sobre la dignificación, la aceptación profunda y el derecho al afecto que todos los seres humanos tienen.

\subsubsection{El tiempo de la acogida}

Tras la herida, tras el acontecimiento traumatizante, la persona debe de ser acogida para reanudar el vínculo afectivo que sirva de guía de desarrollo “(...) la reanudación del vínculo social permite reorganizar la imagen que el herido se hace de sí mismo" (Cyrulnik, 2008, p.214). A veces una única persona es suficiente para avivar la esperanza. Es la primera y más fundamental de las condiciones, es el momento en el que existe la posibilidad de aprender que puede haber amor en su vida, y es esta esperanza la que aviva la "llama" de resiliencia interna, iniciando de manera efectiva el proceso de resiliencia. Si, por el contrario, no existe un encuentro afectivo que dé esperanza de "salir airoso", la llama de la resiliencia se debilita. La sociedad y su cultura, por su parte, dará uno u otro tipo de acogida dependiendo de sus propias características. Esto se transmite no solamente en la calidad del propio acogimiento institucional que la sociedad ofrezca, sino también en el ambiente que se crea en torno a las personas "heridas", que resultará más o menos sensible y más o menos coherente con las necesidades de estas personas.

\subsubsection{El tiempo de la representación}

El proceso de resiliencia es un proceso de transformación de las personas así como de sus acontecimientos vividos. Este proceso se da en el mundo relacional, en el mundo humano; "el proceso de resiliencia permite a un niño herido transformar su magulladura en un organizador del yo, a condición de 
que a su alrededor haya una relación que le permita realizar una metamorfosis" ${ }^{1 "}$ (Cyrulnik, 2008, p.205).

Tras el acogimiento afectivo está nuevamente en el entorno la responsabilidad de continuar con el proceso, ofreciéndole espacios de expresión en los que se encuentren posibilidades de socializar el mundo íntimo, organizar la propia historia y digerir la herida para que, de esta manera, el recuerdo no se imponga y capture la conciencia. Se trata, en definitiva, de facilitar espacios, contextos de expresión de los sentimientos y emociones y cuidar las reacciones para orientar de manera adecuada el proceso.

\subsubsection{El tiempo de la relación}

El tercero de los eslabones de la resiliencia es la relación y el acceso a la cultura social gracias al cual se metamorfosea el sufrimiento. Es sumergirse en la sociedad a través de la cultura. En los dos primeros eslabones es el medio quien abre las puertas, quien posibilita el proceso, ahora el medio lo recibe, el sujeto sobrepasa el umbral y se sumerge en la cultura social donde se puede llevar a cabo esa "reflexión enriquecedora sobre el sentido de la vida", esa "obra útil", esa "obra de arte" y donde "se pueda dar sentido a lo que nos ha ocurrido: organizar la propia historia, comprender y dar" (Cyrulnik, 2008, p.214). Ahora bien, para que la cultura social ofrezca guías de resiliencia es mucho más importante que ofrezca un ambiente que posibilite el surgimiento de actores y no el de meros espectadores. La cultura pasiva no ofrece guías de resiliencia pues lo único que ofrece es el alivio momentáneo otorgado por la satisfacción del consumo que se basa en la diversión o el simple hecho de consumir o poseer. La cultura creativa, sin embargo "es una argamasa social que confiere esperanza a las pruebas de la existencia" (Cyrulnik, 2008, p.197).

\subsection{La afectividad como elemento clave en los procesos de resiliencia}

Más que entre razonamientos, ecuaciones y conocimientos la vida de un ser discurre entre dos universos vitales: el complejo universo de los vínculos interpersonales y el de la propia subjetividad (Goleman, 1995). La afectividad, elemento principal de resiliencia, se desarrolla en el mundo relacional, en las interacciones humanas, es dependiente del entorno humano. La dimensión afectiva, con la vivencia afectiva como núcleo principal, alimenta y da forma al campo cognitivo (Rojas, 1993). Además puede ser responsable directa de nuestros comportamientos y motivaciones (Bisquerra, 2003).

Fue John Bowlby quien, convencido de que los disturbios emocionales en la infancia tenían su origen en las experiencias afectivas vividas, presentó las primeras formulaciones de la teoría del apego entre los años 1958 y 1963. A partir de 1953 Mary Ainsworth, quien fuera la principal colaboradora de Bowlby se dedicó a estudiar mediante investigaciones y experimentos la influencia que ejercía en los niños y niñas el trato afectivo recibido en los hogares (Navarro, 2012).

Las vivencias afectivas adquieren un significado extraordinario y marcan nuestro devenir, siendo el trato afectivo vital desde el inicio de la vida en las personas (Fromm, 2007, p.174). Fue el disfrute de la atención de una persona afectuosa una de las principales características comunes encontradas en la investigación de Werner y Smith (Vanistandael \& Lecomté, 2002), y en los relatos de personas resilientes (Cyrulnik 2008), es el encuentro con un "camarada afectivo" o tutor de resiliencia el principal acontecimiento destacable de su proceso.

El calor humano, el afecto, es la base del desarrollo humano, se vivencia de manera positiva, sí, pero para ello, obviamente, debe ser mostrado y esto puede realizarse por medio de muchas y muy diversas manifestaciones como a través del comportamiento (atento, amable, sincero, tierno, solidario, alegre, etc.), de la expresión corporal (la sonrisa, la mirada, el abrazo, las manos, etc.) o por medio de acciones (la escucha, el regalo, la broma, el halago, el consuelo o las felicitaciones, etc.) (Martí, 2000). En cualquiera de sus formas a través del afecto se valora y dignifica al ser humano y se da pie a su desarrollo íntegro.

Los principales vínculos afectivos se encuentran siempre en el entorno más cercano, la familia, amistades, cónyuge, etc. Pero cuando existen deficiencias afectivas en el entorno de la persona entra en juego "el encuentro" con una persona que pueda avivar la "llama" de la esperanza y convertirse en tutor de resiliencia. Este término es acuñado por Cyrulnik para definir a las persona que nos acompaña de manera incondicional, convirtiéndose en un sostén, administrando confianza e independencia por igual, a lo largo del proceso de resiliencia (Puig \& Rubio, 2015). Pereira (2007) los define como las figuras de apego para dar sentido a lo ocurrido y que generan esperanzas para alcanzar una vida mejor. Este autor propone unas características individuales de los tutores de resiliencia a modo de factores protectores. Tener capacidad para establecer relaciones y vínculos, la creatividad, el sentido del humor, tener iniciativa, autonomía, introspección, sentido moral y confianza en sí mismos son alguno de ellos. Además, añade la capacidad de poner límites claros y permeables que fomenten la autonomía como un factor más en relación con el medio externo. Barudy y Dantagnan (2005) destacan que estos adultos deben ser flexibles, capaces de enfrentar y resolver problemas, con habilidades de comunicación y con destrezas para participar en redes sociales de apego. 


\subsection{Los MENAs y su acogimiento institucional}

Los denominados menores extranjeros no acompañados (a partir de ahora MENAS), son un colectivo dentro del fenómeno migratorio que comenzó a tomar cuerpo en el estado español a mediados de los años 90 y cuyos elementos definidores básicos son, por un lado, la minoría de edad y la condición de extranjero y, por otro, la situación de abandono a instancias legales al no ser requeridos por las familias de origen. En 2009 dos de cada tres menores acogidos provenían del Magreb (Ararteko, 2011). La principal causa migratoria es la económica, pero la situación en origen varía de unos casos a otros (Etxeberria, Murua, Garmendia \& Arrieta, 2012).

Si a la situación de origen se le añade la experiencia del propio viaje en ocasiones traúmatico (Quiroga, Alonso \& Soria, 2009), la entrada en un país extranjero con una lengua y culturas muy distintas, la situación de desvinculación afectiva, así como la propia etapa de desarrollo evolutivo en la que se encuentran, no resulta difícil comprender la importancia de la estimulación de la resiliencia en este colectivo ante estas vivencias que pueden marcar su existencia.

Desde el momento de su llegada, estos menores amparados por diferentes leyes tanto estatales como internacionales (Quiroga, Alonso \& Soria, 2009) quedan a disposición de los servicios sociales competentes que, en el caso del País Vasco, corresponde a las Diputaciones Forales (Etxeberria, Murua, Garmendia \& Arrieta, 2012). Las Diputaciones son, por lo tanto, las encargadas de tutelar a estos menores hasta su mayoría de edad (18 años). Para ello disponen de diferentes centros que son exclusivos para la atención del colectivo y están gestionados por diferentes entidades convenidas con las Diputaciones.

En los centros se trabaja por cubrir sus necesidades básicas y la normalización de su situación, así como en áreas orientadas a su formación y desarrollo personal, aquí es donde entra en juego la figura del educador y la educadora social. Son los educadores y las educadoras sociales las personas que conviven con estos menores en los centros y las encargadas de que, efectivamente, se cubran sus necesidades, desde las más básicas hasta las educativas. Son ellas las responsables de que la convivencia en los centros sea lo más normalizada posible. El educador y la educadora social de estos centros se convierte inevitablemente en un modelo adulto de referencia del entorno que acoge estos menores, es un nexo de unión entre las instituciones, los centros formativos, de salud, etc., y los menores. Es por estas razones también el principal foco de demandas de los menores, lo que hace que, en ocasiones, el educador se sienta un simple medio para los objetivos de éstos (Markez \& Pastor, 2010) y que, a su vez, los menores puedan sentir a estos como desmotivados (Martín \& Coulabi, 2010).
En este trabajo se aborda la resiliencia en la educación social y se concreta en el acogimiento afectivo y la figura del educador y la educadora social en un centro residencial de menores extranjeros. Las preguntas que han guiado este estudio son: ¿̇e estimula la resiliencia en un centro de menores?; ¿reúne el educador y la educadora social de un centro de menores las características y potencialidades de tutor de resiliencia de los menores acogidos?; y ¿̇en qué medida se da y cómo es valorada la afectividad (el trato afectuoso) como instrumento en la intervención del educador y la educadora social en el contexto de un centro de menores extranjeros?, lo que se concreta en los siguientes objetivos:

- Realizar un acercamiento a la noción de resiliencia desde la Educación Social.

- Identificar cualidades de resiliencia en los/as educadores/as del Centro acorde con las características descritas en la literatura de tutores de resiliencia.

- Conocer en qué medida el educador y la educadora social de un centro de menores interviene con las características y potencialidades de un tutor de resiliencia.

\section{Método}

Teniendo en cuenta las características y complejidades subjetivas de la temática a abordar, así como las diferentes interrelaciones entre las personas objeto de estudio y los diferentes contextos, el acercamiento metodológico ha sido cualitativo pretendiendo entender qué se está produciendo y cómo (Sandin, 2003; Torrance, 2012; Tracy, 2010). Lo que justifica este enfoque cualitativo es que el conocimiento no es descubierto sino construido en la propia red social protagonista del estudio y el propósito no es la explicación sino la indagación desde una perspectiva holística esperando amplias y múltiples realidades (Álvarez, 2003).

\subsection{Muestra}

La investigación se ha llevado a cabo en uno de los centros de MENAS de la CAPV elegido por conveniencia, el Centro Residencial de Protección de Menores de Bizkaia Zabaloetxe. Está gestionado por Religiosos Amigonianos- Terciarios Capuchinos y la titularidad es del Instituto Foral de Asistencia Social perteneciente al Departamento de Acción Social de la Diputación Foral de Bizkaia. En la actualidad acoge a 60 menores de procedencia mayoritariamente marroquí divididos en cinco módulos; cada módulo alberga a doce menores y tiene una función singular orientada, sobre todo, por la edad y el comportamiento del menor. 
Esta investigación analiza principalmente la información recogida en el módulo llamado "Albergue" o "Primera acogida" donde se han realizado la mayoría de las observaciones participantes y cinco entrevistas en profundidad. También se han entrevistado educadoras y educadores de otros módulos del Centro Residencial, dos del módulo denominado "Residencia", uno del de "Autonomía" y una de "Preadolescentes", todos ellos y ellas con una media de 7 años de experiencia laboral. Además de la información recabada de las 3 educadoras y 6 educadores, se ha entrevistado al coordinador del centro.

\subsection{Recogida de información e instrumentos}

Las técnicas de recogida de información que se utilizaron durante el periodo de febrero a mayo del 2013 fueron la observación participante y la entrevista en profundidad. A continuación se describen las técnicas y el procedimiento llevado a cabo.

- Durante 12 semanas se utilizó la técnica de observación participante. Marshall y Rossman (1989 en Kawulich, 2006) la definen como "la descripción sistemática de eventos, comportamientos y artefactos en el escenario social elegido para ser estudiado (p.79). Dos días a la semana durante periodos de tres horas aproximadamente se participó en la labor de los educadores en diferentes módulos del Centro. Se recogieron sistemáticamente los datos de cada una de las sesiones referente a la fecha y horas, y posteriormente a la observación se anotaron en un cuaderno de campo las notas relativas a todos los aspectos de la observación relacionados con los objetivos de la investigación.

- Se llevaron a cabo 10 entrevistas en profundidad semiestructuradas con educadores y educadoras, y el coordinador del Centro. Las entrevistas semiestructuradas según Del Rincón y colaboradores (1995) están caracterizadas porque el esquema de preguntas y secuencia no está prefijada, las preguntas son de carácter abierto y el entrevistado tiene que construir la respuesta. Vargas (2011) recomienda en este tipo de entrevistas formular preguntas abiertas, enunciarlas con claridad, únicas, simples y que impliquen una idea principal que refleje el tema central de la investigación. Para la realización de estás se construyó un guión de entrevista con preguntas abiertas generadoras relacionadas con los objetivos y la revisión de la literatura.

Para poder identificar de qué instrumento ha sido extraída la información se han utilizado los siguientes códigos:

- Notas de campo de la observación participante (OP).
- Entrevista semiestructurada al coordinador del Centro (Coor).

- Entrevistas en profundidad a los educadores y las educadoras del Centro (Edu1; Edu2;...; Eduq).

\subsection{Procedimiento}

El análisis de la información recogida, se ha realizado siguiendo un proceso inductivo de las informaciones aportadas por las transcripciones de las entrevistas y notas de campo, a través de un sistema categorial emergente. Este proceso ha facilitado la extracción de las ideas principales de la información, organizándola en categorías y subcategorías (Rodríguez, Gil \& García 1996).

\section{Análisis e interpretación de los resultados}

Tras analizar y sintetizar la información recogida los resultados muestran evidencias de: detección de factores de riesgo y protección; características de la intervención educativa en tiempo de acogida; la afectividad y el ambiente como claves en el tiempo de representación y relación; y bases socioeducativas de la acción tutorial resiliente.

\subsection{Detección de factores de riesgo y protección del proceso de resiliencia en MENAS}

Los testimonios de los educadores y educadoras presentan que la mayoría de MENAS vive una experiencia dura que tiene un gran impacto en su proceso vital, lo que se podría identificar como herida de la experiencia migratoria.

"Es muy dura para ellos y sus familias, supone desarraigo, ruptura, sacrificio, riesgo" (Edut).

"Un choque, más de lo que pueden imaginar en un momento..." (Edu2). "...es una edad en la que se necesita mucho apoyo [...] llegan a una cultura distinta [...] es un gran cambio" $(O P)$.

Los propios menores normalmente evitan hablar de ello y cuando lo hacen no suelen mostrarse muy emotivos.

“...algunos te cuentan su experiencia [...] que han estado en los bajos de un camión [...] y cuando salieron no podían mover las piernas [...] y te lo cuentan incluso riéndose [...] y tú piensas en lo que eso supone [...], te dicen de repente que llevan tres años sin ver a su familia [...] y tú piensas, ijo!, una madre es una madre" (Eduz).

La exigencia de las necesidades prioritarias a cubrir deja en un segundo lugar la propia experiencia migratoria, al respecto es interesante observar cómo al- 
gunos educadores ante la pregunta de cómo ven la experiencia migratoria a su edad contemplan como más significativo el hecho de lograr o no los objetivos que les ha hecho emigrar que la experiencia en sí.

“...la experiencia es traumatizante para el que fracasa [...] el hecho de no triunfar, vienen con un proyecto familiar para lograr el éxito económico..." (Edu4).

La información recogida muestra también las referencias afectivas como "andamio" de los menores $y$, por lo tanto, como factor de protección del posible proceso de resiliencia. Se destacan tres figuras principales: los amigos (compañeros del centro mayoritariamente), la familia de origen y los educadores; aunque la mayoría considera a la familia de origen como una referencia afectiva significativa y que sigue siendo muy influyente en el menor.

“... a pesar de los problemas los vínculos con la familia siguen siendo muy fuerte..." (OP).

"... cuando tienen que tomar decisiones importantes siempre llaman a la familia [...] pero suelen destacar lo efímero de los contactos y la poca profundización de los mismos" (Edu5).

Los compañeros son considerados como una de las principales fuentes de apoyo, por lo tanto suelen mostrar un gran apego hacia amigos concretos y comportamientos bien diferenciados para con los educadores.

“... ellos se apoyan mucho entre ellos, sobre todo si son del mismo pueblo..." (Eduz).

"... puedes apreciar que alguno es cercano con los amigos pero distante con los educadores..." (OP).

Las personas educadoras se ven como un apoyo importante, pero son pocas las que se ponen en primer lugar como referencia afectiva, aunque reconocen que en ocasiones, se convierten en personas muy significativas para los menores.

“... en los más pequeños, sobre todo, ves que el educador es un referente muy importante..." (Edu6).

“... los educadores somos referentes especiales [...] también los profesores de los centros que algunos son verdaderos educadores y se convierten en referente [...], suplir a la familia es muy difícil..." (Edu5).

En algunos casos incluso conservan amistades de menores que pasaron por el centro y reconocen haber ayudado a muchos. Expresan que mientras están en el centro los menores les ven como "unos pesados que siempre están encima" (Eduz) pero que cuando salen y pasa un tiempo la mayoría valora positivamente tanto la estancia en el centro como el trato recibido.

\subsection{Características de la intervención educativa en el tiempo de acogida}

Para que en los centros se de la primera fase de acogida, una de las más importantes en el proceso de resiliencia, es necesario un primer acercamiento por parte de las educadoras y educadores, que en muchas ocasiones es definido como dificultoso.

“...no se abren, tienes que sacarles las cosas con calzador..." (Edu6).

En la mayoría de los casos sienten que generalizar es difícil y que depende mucho de cada caso, pero coinciden en expresar, que es un colectivo que no se abre mucho y que además, la visión generalizada de la persona educadora, como un medio más para sus fines, influye en la calidad del acercamiento.

“...tienes que ser tú el que se abra [...] con el tiempo y la cercanía te pueden contar sus experiencias, sus vivencias, pero es muy difícil que te expresen sus sentimientos..." (OP).

"...el trato es solo de lo del día a día, el curso, la ropa, la comida [...] te cuentan, pero no cómo se sienten" (Edu3).

Sin embargo, otras personas educadoras consideran que es bastante factible el que se abran emocionalmente.

“...cuando ves que están mal o que le ha pasado algo a su familia, te acercas y les preguntas [...] yo nunca me he encontrado el caso de que no quieran hablar" (Eduq).

"Tampoco hay mucha opción, están los compañeros que nos son referentes [...] los padres que no están físicamente y hay unos roles establecidos [...] y nosotros, que estamos en medio, somos el tío lejano, no hay otra salida [...] nos pueden contar historias y a la vez saben que no les vamos a currar [...] son ellos mismos los que se acercan [...] no me siento padre ni colega estamos en medio [...] no dejas de ser un tío que no es su padre, que habla otro idioma..." (Edu2).

\subsection{La afectividad y el ambiente, claves en el tiempo de representación y relación}

Todos las educadoras y educadores remarcan la importancia de la afectividad en su trabajo, y algunos la ven como el elemento clave para establecer una relación educativa de calidad.

“...es muy importante, cuando llegan tienen un vacío tremendo, el educador es una pieza clave en ese momento [...] hay que ser afectivo" (Edu8).

"Es fundamental, es el principal instrumento con que cuenta el educador para ejercer su misión. Si el edu- 
cador no llega al corazón del educando, no le puede ayudar a crecer y desarrollarse" (Coor).

"...es vital, la base de todo..." (Edu8).

"Esencial, tiene que sentirse apreciado querido, comprendido y acompañado como con los padres..." (Edu6).

Un aspecto importante destacado en las diferentes entrevistas, es que en el trabajo del tutor $y$ la tutora para que se dé un trato afectivo de calidad, es necesario estar a gusto con la labor a realizar, ya que de lo contrario se puede reducir a lo puramente asistencial.

“...si estar a gusto es importante en cualquier oficio en este mucho más porque trabajamos con personas..." $(\mathrm{OP})$.

“...es muy importante, ellos lo notan en el ambiente [...] si estás a disgusto no te vas a dedicar a ellos como debieras [...] vas a 'abrir puerta y cerrar puerta' y punto..." (Eduz).

Tomarse la labor cotidiana con ilusión depende, según las educadoras, del ambiente que se respire en el centro y, en el propio módulo. Los y las educadoras destacan tres aspectos que influyen en este ambiente que si es adecuado favorecerá una acción tutorial resiliente: el servicio institucional, los compañeros de trabajo y el perfil del menor.

\subsection{Paciencia, límites y escucha, bases socioe- ducativas de la acción tutorial resiliente}

Los aspectos que más se mencionan se observan en la relación cotidiana con los menores, y son la paciencia y trabajar los límites, ambos, cuando no se dan en un contexto estrictamente de contención (lo que sucede en no pocas ocasiones), están orientados al logro de objetivos educativos. En la mayoría de las situaciones es apreciable todo un entramado de valores, aceptación de la persona, dedicación y confianza en el desarrollo del menor al que se le dedica tiempo y atención, pilares de la resiliencia.

\footnotetext{
"Aquí sacamos un máster en paciencia" (OP).

“... todo es tiempo, tiempo para realizar un acercamiento cercano, tiempo para que asimilen muchas cosas, para que maduren [...] ser paciente tiene sus recompensas, no siempre [...] pero tampoco queda otra..." (OP).

“... insistes e insistes y estás encima y al final ves los resultados pero requiere de mucha constancia..."(OP).
}

Saber poner límites requiere esfuerzo y está muy ligado a la paciencia y generalmente requiere enfrentarse a un comportamiento resultado de falta de habilidades sociales, comunicación, convivencia o valores humanos.
“... podemos hacer como que no vemos nada pero entonces perdemos la oportunidad de que aprendan ciertos valores..." (OP).

“...cuanto más tolerancia ante esos comportamientos peor, la hostia que se dan cuando salen de aquí es más gorda [...] si procuramos su bien debemos de evitar esto..." (OP).

"En líneas generales se dedica atención y se muestra interés por la persona [...] yo me dedico a ellos, me esfuerzo, podría venir aquí y pasar de todo, hacer como que no veo ciertas cosas, pero este es mi trabajo..." (OP).

Las muestras de afecto que se pueden observar al cabo del día son muy variadas: el saludo, las muestras de interés por su estado, los mensajes de ánimo, las acciones de acompañamiento, la intención de hacer reír, los mensajes y las valoraciones positivas de actos $o$ actitudes y, en relación al segundo "eslabón" de resiliencia, la disposición a la escucha.

“... tienen necesidades emocionales [...] les preguntas que tal la familia o que tal tu hermano y te responden bien, bien, pero no quieren ir más allá, luego ves que se encierra en el cuarto y está con algún compañero, depende mucho de la confianza que haya..." (OP).

"... percibes que tiene la necesidad de hablar de soltarse y tú te dispones a oírle y le muestras que ahívas a estar [...] yo dedico mucho tiempo a intentar que hablen y escucharles, me parece muy importante..." (Coor).

\section{Discusión y conclusiones}

Desde la educación social la resiliencia nos sugiere una actitud responsable por la importancia que tiene la calidad de interacción de las personas para el desarrollo humano; y optimista pues es algo estimulable tanto antes como después de los infortunios de la vida. Desde cualquier ámbito de la educación social hay espacio para estimular este proceso porque se centra en y desde el entorno humano, se interactúa y hay una relación constante y estrecha con personas. La noción de resiliencia supone un paso más a la inalcanzable receta mágica de la felicidad y la superación de las desgracias del ser humano y esto aporta criterio y fundamentación a un elemento esencial de la educación social como es la esperanza frente a supuestas determinaciones. No se trata de atribuir funciones terapéuticas a la educadora y el educador social sino de comprender que la resiliencia se entrelaza con la educación al ser también un proceso afectivo y comunicativo.

La mejora de la sociedad y el desarrollo íntegro de las personas son el principal fin de la educación social y no son pocos los ámbitos de ésta en los que se interactúa con personas que han pasado o pasan por serias adversidades ya sea en el plano social, familiar o personal. La educadora y el educador social, en la 
medida que interactúa y forma parte de ese entorno humano donde se desarrolla el proceso de resiliencia, se encuentra en un contexto donde puede aceptar, dignificar y dar afecto a las personas. Los testimonios recogidos de educadores en esta investigación corroboran aquellas investigaciones que han puesto de manifiesto la gran importancia que juegan las personas afectuosas por las que son tratadas las personas que han pasado por grandes dificultades (Vanistandael \& Lecomté, 2002) y el gran valor que puede tener en el proceso, en este caso de los MENAS, el encontrarse con una persona que les acoge y trate con afecto (Cyrulnik 2008).

Dentro del trato afectivo se incluye la necesidad de autoridad y límites por parte de las personas educadoras. Estos resultados coinciden con el modelo de la rueda de la resiliencia planteado por Henderson y Milstein (2003) que ha obtenido resultados exitosos con jóvenes en el ámbito escolar (Barranco, 2008). Está compuesto por seis factores, entre los cuales no son solamente necesarios los orientados a la construcción de la resiliencia (brindar afecto y apoyo, establecer y transmitir expectativas elevadas y brindar oportunidades y participación significativa), sino también como en el caso de poner límites a mitigar los factores de riesgo (fijar límites claros y firmes, enriquecer los vínculos prosociales y enseñar "habilidades para la vida"). A través de esta actitud, como se puede ver en este estudio, se le manda un mensaje al menor: te recibo y te acepto pero rechazo tus comportamientos inadecuados y así te lo muestro porque mi interés es que te desarrolles como persona.

El educador y la educadora social de un Centro de Menores Extranjeros no Acompañados no suple a la familia, pero en la medida en que convive con el menor y es responsable de su desarrollo adquiere un rol similar, debido a las exigencias de convivencia y educativas. A tenor de las entrevistas realizadas se observa que los educadores y educadoras, junto a otros agentes como la familia o miembros del grupo de iguales, forman parte de esas personas que acompañan y apoyan el proceso de afrontar la situación de fuerte vulnerabilidad y crisis; que proporcionan una relación mediante la cual los menores pueden recuperar o avivar la esperanza de afrontar la situación que viven (Cyrulnik, 2001). Se crea un vínculo educativo en el que existe una interacción estrecha, de lo que resulta un contexto adecuado no solo para el desarrollo de la persona y sus valores sino para estimular la resiliencia y que el educador y la educadora puedan ser tutores de la misma. A través de la atención, la dedicación y el interés que se muestra se reconoce a estos menores, se les valoriza y se les dignifica, ahora bien, existe el peligro de que este acogimiento afectivo pierda calidad a medida que se van incrementando algunos condicionantes de tipo institucional, organizacional o de perfil de los educandos, pudiendo llegar a convertirse en meramente asistencial y normativo.

En ocasiones aunque los educadores no conozcan el concepto, salen a la luz muchos elementos de resiliencia a los que puede dar pie una intervención socioeducativa.

“... conmigo habla mucho, me cuenta muchas cosas [...] vivía en la calle desde muy pequeño [...] la policía cada vez que le cogía en la frontera le daba unas hostias, así lo cuenta [...] en Marruecos ha estado en más de un centro y allí no es como aquí [...] les tratan muy mal, son como cárceles [...] ha sufrido mucho [...] pero se porta bien [...] no me explico cómo puede ser tan noble [...] a veces cuando vamos a comer les digo: ivenga! vamos al comedor como en el centro de Tánger y se ponen en fila con las manos en los hombros del compañero que tienen delante y comienzan andar [...] pero lo hacemos para reírnos..." (OP).

Se encuentra una aceptación de la persona y un acogimiento afectivo que se muestra a través del interés y la escucha. Como describe Cyrulnik (2008) hay necesarios momentos de expresión de lo vivido y oportunidades de metamorfosear el sufrimiento a través del sentido del humor, de lo que puede resultar el avivamiento de la esperanza, de la llama de la resiliencia. En este sentido, una de las conclusiones de este trabajo de investigación es que los Centros de Acogida, aunque no expliciten el concepto de resiliencia en el proyecto educativo, son un lugar inmejorable para estimularla, puesto que es el principal apoyo que recibe este colectivo desde su llegada hasta la edad adulta; se atiende a sus principales necesidades para afrontar el futuro y existe una disposición a la ayuda constante. Sin embargo, será el tiempo lo que determine si la experiencia de los menores en el centro ha sido significativa y ha servido para avivar la llama de la resiliencia.

Por último, a pesar de las limitaciones de esta investigación por su muestra y carácter cualitativo que no permite extrapolar los resultados y plantea la necesidad de nuevos trabajos en este camino, sí que nos muestra que esta interesante y no muy desarrollada temática de la resiliencia tiene una gran importancia en el trabajo socioeducativo y la Pedagogía Social. Por ello, se considera importante continuar transitando este impredecible y necesario viaje, complementando a través de investigaciones aplicadas aspectos que no se han abordado como la mirada del colectivo que ha pasado por estos centros, la realidad de otros centros de menores o la relación con otros colectivos y ámbitos de la Educación Social. 


\section{Referencias bibliográficas}

Aguirre, A. (2002). Capacidad y factores asociados a la resiliencia, en adolescentes del CE Mariscal Andrés Avelino Cáceres del Sector IV de Pamplona Alta San Juan de Miraflores (Doctoral dissertation, Tesis de Licenciatura no publicada). Lima, Perú: Facultad de Medicina Humana de la Universidad Nacional Mayor de San Marcos.

Álvarez, J. (2003). Cómo hacer investigación cualitativa: Fundamentos y Metodología. México: Paidós.

Ararteko (2011). Infancias vulnerables. Informe extraordinario. Retrieved from http://argitalpen.ararteko.net/index.php? leng=cast\&id_l=45

Barudy, J., \& Dantagnan, M. (2005). Los buenos tratos a la infancia Parentalidad, apego y resiliencia. Barcelona: Gedisa.

Becoña, E. (2006). Resiliencia: definición, características y utilidad del concepto. Revista de psicopatología y psicología clínica, $11(3), 125-146$.

Barranco, C. (2009). Trabajo Social, calidad de vida y estrategias resilientes. Portularia: Revista de Trabajo Social, 9, $133-145$.

Bisquerra, R. (2003). Educación emocional y competencias básicas para la vida. Investigación Educativa (RIE), 21(7), 7-43.

Chukri, M. (2000). El pan desnudo. Madrid: Debate.

Cyrulnik, B. (2008). Los patitos feos: La resiliencia: una infancia infeliz no determina la vida. Barcelona: Gedisa.

Del Rincón, D., Arnal, J., Latorre, A., \& Sans, A. (1995). Técnicas de Investigación en Ciencias Sociales. Madrid: Dykinson

Drapeau, S., Saint-Jacques, M. C., Lepine, R., Begin, G., \& Bernard, M. (2007). Processes that contribute to resilience among youth in foster care. Journal of adolescence, 30(6), 977-999.

Etxeberria, F., Murua, H., Garmendia, J., \& Arrieta, E. (2012). Menores Inmigrantes No Acompañados (MENA) en Euskadi y Aquitania: elaboración y puesta en marcha de un Plan de Formación para Educadores/as y Responsables. Revista de educación social (RES), 15. 1-28.

Fromm, E. (2007). El arte de amar. Barcelona: Paidos.

Fuentes, N., Medina, J., Van Barneveld, H., \& Escobar, S. (2009). Resiliencia y salud en niños y adolescentes. Ciencia Ergo Sum, 16(3), 247-253

Goleman, D. (1996). Inteligencia emocional. Barcelona: Kairos.

Grotberg, E.H. (2001). Nuevas Tendencias en Resiliencia. en Melillo, A. \& y Suárez, E. (Comp.). Resiliencia. Descubriendo las propias resiliencias. Barcelona: Paidós.

Henderson, N., \& Milstein, M. M. (2003). Resiliency in schools: Making it happenfor students and educators. Thousand Oaks, CA: Corwin.

Kawulich, B. (2006). La observación participante como método de recolección de datos Forum Qualitative Sozialforschung/ Forum: Qualitative Social Research 6(2), Art. 43, Retrieved from http://nbn-resolving.de/urn:nbn:de:0114-fqsO5O2430

Kotliarenco, M. A., Cáceres, I., \& Fontecilla, M. (1997). Estado de arte en resiliencia. Washington DC: Organización Panamericana de la Salud.

López, D., \& García, E. (2010). Sistemas de protección emergentes: el caso de Tánger, ciudad fronteriza. Pedagogía social: revista interuniversitaria, 17, 57-70.

Margalit, M., \& Idan, O. (2004). Resilience and hope theory: An expanded paradigm for learning disabilities research. Thalamus, 22(1), 58-64.

Markez, l., \& Pastor, F. (2010). Menores Extranjeros No Acompañados (MENAs), un colectivo especialmente vulnerable ante las drogas. Zerbitzuan, 48, 71-85.

Marshall, C., \& Rossman, B. (1989). Designing qualitative research. Newbury Park, CA: Sage.

Martí, M. A. (2000). La afectividad. Los afectos son la sonrisa del corazón. Madrid: E.I.U.

Martín, Z., \& Coulaby, A (2010). Situación de los centros de acogida temporal para menores extranjeros no acompañados. In C. Manzanos Bilbao (2010). ¿Menores sin derechos?: infancia extranjera desprotegida, juventud penalizada. Eskubiderik gabeko adingabeak?: babesik gabeko haur atzerritarrak, gazteria zigortua. Gasteiz: Ikusbide.

Munist, M., Santos, H., Kotliarenco, M. A., Suárez Ojeda, en Infante, F., \& Grotberg (1998). Manual de identificación y promoción de la resiliencia en niños y adolescentes. Washington, DC: Organización Panamericana de la Salud. Organización mundial de la Salud.

Munist, M., Suárez, N., \& Suarez, B. (2010) Resiliencia en los adolescentes. In D. Pasqualini, \& A. Llorens (Comp.) Salud y bienestar de los adolescentes y jóvenes: una mirada integral. Buenos Aires: Universidad de Buenos Aires, Facultad de Medicina.

Muñoz, V., \& De Pedro, F. (2005). Educar para la resiliencia. Un cambio de mirada en la prevención de situaciones de riesgo social. Revista complutense de educación, 16(1), 107-124.

Muñoz-Silva, A. (2012). El estudio de la resiliencia desde la perspectiva evolutiva y su aportación a la comprensión del riesgo y la protección en la intervención social. Portularia, Revista de Trabajo Social, 12(1), 9-16.

Navarro, M. (2012). El apego y la seguridad emocional en la educación infantil. Proyecto Fin de Grado no publicado, Universidad internacional de la Rioja. 
O'Dougherty, M., Masten, A. S., \& Narayan, A. J. (2013). Resilience processes in development: Four waves of research on positive adaptation in the context of adversity. In S. Goldstein \& R. B. Brooks (Eds.), Handbook of resilience in children (pp. 1538). New York: Springer.

Oliva, A. (2004). Estado actual de la teoría del apego. Revista de Psiquiatría y Psicología del Niño y del Adolescente, 4(1), 65-81.

Paskual, J. (2010). Aproximación sociológica a la función del educador de menores: El caso práctico de los centros de acogida y hogares funcionales de menores extranjeros no acompañados en el territorio histórico de Bizkaia. In Manzanos C. Bilbao (2010). ¿Menores sin derechos?: infancia extranjera desprotegida, juventud penalizada. Eskubiderik gabeko adingabeak?: babesik gabeko haur atzerritarrak, gazteria zigortua. (pp. 125-132). Gasteiz: Ikusbide.

Pereira, R. (2007). Resiliencia individual, familiar y social. Interpsiquis. Octavo congreso virtual de psiquiatría.

Poseck, V., Baquero, B. C., \& Jiménez, M. L. V. (2006). La experiencia traumática desde la psicología positiva: resiliencia y crecimiento postraumático. Papeles del psicólogo, 27(1), 40-49.

Puig, G., \& Rubio, J. L. (2015). Tutores de resiliencia: Dame un punto de apoyo y moveré MI mundo. Barcelona: Gedisa.

Quiroga, V., Alonso, A. y Soria, M. (2010). Sueños de bolsillo: menores migrantes no acompañados/as en el País Vasco. Administración de la comunidad autónoma del País vasco, Departamento de empleo y asuntos sociales. Vitoria-Gasteiz: S.P.C.G.V.

Reich, J. W., Zautra, A. J., \& Hall, J. S. (Eds.). (2010). Handbook of adult resilience. New York: Guilford Press.

Rodríguez, G., Gil, J., \& García, E. (1996). Metodología de la investigación cualitativa. Málaga: Aljibe.

Rojas, E. (1987). El laberinto de la afectividad. Madrid: Espasa-Calpe.

Sandín, M. (2003). Investigación cualitativa en Educación: fundamentos y tradiciones. Madrid: McGraw-Hill/Interamericana.

Torrance, H. (2012). Triangulation, Respondent Validation, and democratic participation in mixed Methods Research. Journal of Mixed Methods Research, 6, 111-123.

Tracy, S. (2010). Qualitative Quality: Eight “Big-Tent” Criteria for Excellent Qualitative Research. Qualitative Inquiry, 16, 837851.

Ungar, M. (2004). A constructionist discourse on resilience multiple contexts, multiple realities among at-risk children and youth. Youth \& society, 35(3), 341-365.

Vargas, I (2012). La entrevista en la investigación cualitativa: nuevas tendencias y retos. Calidad en la Educación Superior, 3(1), $119-139$.

Vanistandael, S., \& Lecomte, J. (2002). La felicidad es posible. Despertar en niños maltratados la confianza en sí mismos: construir la resiliencia. Barcelona: Gedisa.

Villalba, C. (2003). El concepto de resiliencia individual y familiar. Aplicaciones en la intervención social. Intervención Psicosocial, 12(3), 283-299.

Villalba, C. (2011). El enfoque de resiliencia en Trabajo Social. Acciones e Investigaciones sociales, 1, 466-489.

Walsh, F. (2012). Facilitating family resilience: Relational resources for positive youth development in conditions of adversity. In M. Unger (Ed.), The social ecology of resilience: A handbook of theory and practice (pp. 173-185). New York: Springer.

Werner, E. (2007). How children become resilient: Observations and cautions. In N. Henderson (Ed.) Resiliency in action: Practical ideas for overcoming risks and building strengths in youth, families, and communities (pp. 15-23). Solvang, CA: Resiliency $\ln$ Action.

\section{Notas}

${ }^{1}$ Cyrulnik enuncia en numerosas ocasiones el término "metamorfosis", de hecho, realiza en su obra una comparativa del proceso de resiliencia de los seres humanos desde su nacimiento hasta la edad adulta con el proceso del ciclo de vida de la mariposa que pasa de oruga inmóvil a ser alado, a mariposa. Cyrulnik, como otros autores, expresa que el término resiliencia implica también energía y creatividad a raíz del sufrimiento por lo que debiera significar más que adaptación o supervivencia. 


\section{CÓMO CITAR ESTE ARTÍ́CULO}

Martín, J. M., V., Alonso, I., \& Tresserras, A. (2016). Aportaciones del paradigma de resiliencia a la acción socioeducativa. El caso del Centro de menores extranjeros no acompañados Zabaloetxe. Pedagogía Social. Revista Interuniversitaria, 28 157-168. DOI:10.7179/PSRI_2016.28.12

\section{DIRECCIÓN DE LOS AUTORES}

José Miguel Martín de Castro: Universidad del Pais Vasco / Euskal Herriko Unibertsitatea

Israel Alonso Sáez. Universidad del Pais Vasco / Euskal Herriko Unibertsitatea. E-Mail: israel.alonso@ehu.es

Alaitz Tresserras Angulo. Universidad del Pais Vasco / Euskal Herriko Unibertsitatea. E-mail: alaitz.tresserras@ehu.es

\section{PERFIL ACADÉMICO}

José Miguel Martín de Castro: Graduado en Educación Social en la Escuela Universitaria de Magisterio de Bilbao de la Universidad del País Vasco. Educador Social con experiencia en el ámbito de los Menores Extranjeros no acompañados. Ha trabajado en el Centro de protección de menores Zabaloetxe en Lujua (Vizcaya), gestionado por Amigonianos (Capuchinos, Franciscanos) y cuya titularidad es del Instituto Foral de Asistencia Social y en un Centro de Urgencia gestionado por Nuevo Futuro en Gasteiz.

Israel Alonso Sáez. Doctor en Pedagogía por la Universidad del Pais Vasco/ Euskal Herriko Unibertsitatea (UPV/EHU). Educador Social y Pedagogo. Profesor del Departamento de Didáctica y Organización escolar en el Grado de Educación Social en la UPV/EHU. Ha impartido docencia en direntes Masters como el de Modelos y Estrategias de acción educativa y social en la infancia y adolescencia de la Universidad Ramon Llul y el de participación y desarrollo comunitario en la Universidad del País Vasco. Vinculado a la práctica e investigación relacionada con la intervención socioeducativa con personas en situación de exclusión y en estos momentos al cambio e innovación en la Universidad.

Alaitz Tresserras Angulo. Profesora del Departamento de Didáctica y Organización Escolar de la Universidad del País Vasco en los Grados de Educación Social y Educación Primaria en la Escuela Universitaria de Magisterio de Bilbao. Licenciada en pedagogía social, psicodramatista, orientadora familiar y sexual y antigua técnica del Consejo de la Juventud de Euskadi. Actualmente realiza su trabajo doctoral e investigación científica en el ámbito de la psicodidáctica, poniendo su atención en metodologías de construcción de conocimiento colectivo, y en metodologías de formación de formadores que posibilten el desarrollo profesional de la identidad individual y colectiva. 